\title{
MORTALIDADE MATERNA DE MULHERES NEGRAS: PERFIL EPIDEMIOLÓGICO EM ALAGOAS
}

\author{
MATERNAL MORTALITY OF BLACK WOMEN: EPIDEMIOLOGICAL PROFILE \\ IN ALAGOAS
}

\author{
MORTALIDAD MATERNA DE MUJERES NEGRAS: PERFIL EPIDEMIOLÓGICO EM ALAGOAS
}

Jorge Luis de Souza Riscado ${ }^{1}$, Nathália Christina Lopes Flores ${ }^{2}$, Jairo Calado Cavalcante ${ }^{3}$, Yanne Carolline Silva Mesquita ${ }^{4}$, Maria das Graças M. M. Taveira ${ }^{5}$, Laudemi José de Oliveira6

\section{RESUMO}

Objetivo: a morte de uma mulher na gestação é um importante indicador de saúde da população feminina. Objetivou-se realizar pesquisa quantitativa com a análise da mortalidade materna dos anos de 2006 a 2015. Método: para o cálculo da taxa de mortalidade materna, utilizaram-se os bancos de dados SIM e SINASC, selecionando as cores/raças e gravidez, parto e puerpério. Foram confeccionados gráficos por meio do programa Excel e mapas de Alagoas, ilustrando os resultados, com o programa TABWIN. Também foi realizada análise de razão de chances por meio de cálculo odds ratio. Resultados: em 2010, Alagoas apresentou população composta por (31\%) brancas, $(8 \%)$ pretas e (61\%) pardas (população negra de 69\%). Em comparação a essas porcentagens, as mortes maternas por raça/cor no ano de 2010 foram de $17 \%$ nas brancas, $9 \%$ nas pretas e $74 \%$ nas pardas ( $83 \%$ negras). Apesar disso, quando estimada a constituição racial do Estado nos dez anos a partir dos nascidos vivos, conclui-se população de $(12 \%)$ brancas e negras $(88 \%)$, sendo $1 \%$ pretas e $87 \%$ pardas, enquanto a porcentagem de mortes para o mesmo período foi de $15 \%$ brancas, $80 \%$ pardas e $5 \%$ pretas ( $85 \%$ negras). No cálculo do risco de morte pelo odds ratio no segmento de cada raça/cor, as brancas obtiveram 0,359; pardas, 1,591 e negras, 1,561. Conclusão: a cor preta, neste estudo, teve associação não significativa. Em relação à escolaridade, a morte das negras sempre tem elevados patamares, principalmente quando analisados os menores índices de escolaridade. Quanto maior a escolaridade, menores os índices de mortalidade. A $2^{a}$ Região de Saúde de Alagoas apresentou as maiores taxas de mortalidade em quatro anos: 2007, 2011, 2012 e 2014. Os números no Estado de Alagoas são bastante alarmantes em um pacto que reverbera em situação de saúde pública.

\footnotetext{
${ }^{1}$ Doutor. Universidade Federal de Alagoas. Maceió ( $\left.A L\right)$, Brasil.

$2,4,6$ Graduandos. Universidade Federal de Alagoas. Maceió (AL), Brasil.

${ }^{3}$ Especialista. Universidade Federal de Alagoas. Maceió ( $\left.A L\right)$, Brasil.

${ }^{5}$ Mestre. Universidade Federal de Alagoas. Maceió (AL), Brasil.
} 
Palavras-chave: Grupos de Populações Continentais; Mortalidade Materna; Análise Quantitativa.

\begin{abstract}
Objective: The death of a woman in pregnancy is an important indicator of the health of the female population. The objective was to conduct a quantitative research with the analysis of maternal mortality from 2006 to 2015. Method: To calculate the maternal mortality rate, we used the SIM and SINASC databases, selecting the colors / races and pregnancy, childbirth and the puerperium. Charts were made using the Excel program and maps of Alagoas, illustrating the results, with the TABWIN program. Odds ratio analysis was also performed by calculating odds ratios. Results: In 2010, Alagoas had a population composed of $(31 \%)$ white, $(8 \%)$ black and (61\%) brown (black population $69 \%)$. Compared to these percentages, maternal deaths by race / color in 2010 were $17 \%$ in white, $9 \%$ in black and $74 \%$ in brown ( $83 \%$ black). Nevertheless, when the racial constitution of the state is estimated in the ten years from live births, a population of $(12 \%)$ white and black ( $88 \%)$ is concluded, being $1 \%$ black and $87 \%$ brown, while the percentage of deaths for the same period it was $15 \%$ white, $80 \%$ brown and $5 \%$ black ( $85 \%$ black). In calculating the risk of death by odds ratio, in the segment of each race / color, white obtained 0.359; brown, 1,591 and black, 1,561. Conclusion: Black color in this study had no significant association. Regarding education, the death of black women always has high levels, especially when analyzing the lowest levels of education. The higher the education, the lower the mortality rates. The 2nd Alagoas Health Region had the highest mortality rates in four years: 2007, 2011, 2012 and 2014. The figures in the State of Alagoas are quite alarming in a pact that reverberates in public health
\end{abstract}

Keywords: Continental Population Groups; Maternal Mortality; Quantitative Analysis.

\title{
RESUMEN
}

Objetivo: la muerte de una mujer en el embarazo es un importante indicador de salud de la población femenina. Se objetivó realizar investigación cuantitativa, con el análisis de la mortalidad materna de los años 2006 a 2015. Método: para el cálculo de la tasa de mortalidad materna se utilizaron los bancos de datos SÍ y SINASC, seleccionando los colores / razas y el embarazo, parto y el puerperio. Se han confeccionado gráficos a través del programa Excel y mapas de Alagoas, ilustrando los resultados, con el programa TABWIN. También se realizó un análisis de la razón de las probabilidades a través de cálculos odds ratio. Resultados: en 2010, Alagoas presentó población compuesta por (31\%) blancas, ( $8 \%)$ negras y $(61 \%)$ pardas (población negra de $69 \%)$. En comparación con estos porcentajes, las muertes maternas por raza/color en el año 2010 fueron del $17 \%$ en las blancas, el $9 \%$ en las negras y el $74 \%$ en las pardas ( $83 \%$ negras). A pesar de ello, cuando se estima la constitución racial del Estado en los diez años a partir de los nacidos vivos, se concluye una población de (12\%) blancas y las negras ( $88 \%)$ siendo $1 \%$ negras y $87 \%$ pardas, mientras que el porcentaje de muertes para el mismo período fue de $15 \%$ blancas, $80 \%$ pardas y $5 \%$ negras ( $85 \%$ negras). En el cálculo del riesgo de muerte por el odds ratio. en el segmento de cada raza/color, las blancas obtuvieron 0,359; pardas, 1,591 y negras, 1,561. Conclusión: el color negro, en este estudio, tuvo una asociación no significativa. En relación a la escolaridad, la muerte de las negras siempre tiene elevados niveles comparativos, principalmente cuando se analizan los menores índices de escolaridad. Cuanto mayor nivel educativo, menor el índice de mortalidad. La $2^{\text {a }}$ Región de Salud de Alagoas presentó las mayores tasas de mortalidad en cuatro años: 2007, 2011, 2012 y 2014 . Las cifras en el Estado de Alagoas son bastante alarmantes en un pacto que reverbera en situación de salud pública. 
Palabras clave: Grupos de Población Continentales; Mortalidad Materna; Análisis Cuantitativo.

\section{INTRODUÇÃO}

Paulatinamente, o Brasil tem avançado em algumas melhorias da atenção ao parto e ao nascimento frutos de uma série de esforços e iniciativas governamentais e da sociedade. Porém, a redução da morbimortalidade materna e infantil permanece um desafio. Ainda que o acesso ao pré-natal seja praticamente universal na perspectiva do SUS, a qualidade dessa atenção ainda requer muitas melhorias.

Embora no Brasil, em 2011, tenha sido implantada a "Rede Cegonha", enquanto estratégia do Ministério da Saúde, objetivando, por si, uma rede de cuidados para garantir às mulheres o direito ao planejamento reprodutivo e atenção humanizada à gestação e, às crianças, o direito ao nascimento seguro e ao crescimento e desenvolvimento saudáveis, ainda se percebem uma fragilidade de ações e tomada de atitude por parte programática dos governos.

As mulheres negras não possuem um atendimento integral e equitativo que prioriza o SUS ${ }^{1}$. Ainda, outros fatores genéticos, como predisposição a patologias, diabetes e hipertensão arterial, contribuem para o maior risco da gestação nas mulheres negras².

A ocorrência da morte materna representa violação dos direitos humanos das mulheres e reflete a falta de acesso aos serviços de saúde e as condições socioeconômicas desfavoráveis. Ao considerar que a mortalidade materna é evitável em até $92 \%$ dos casos, deve-se dar grande importância ao monitoramento dos índices de forma a favorecer o redirecionamento de políticas de saúde.

Com base nisso, a redução da mortalidade materna passou a ser $05^{\circ}$ Objetivo de Desenvolvimento do Milênio (ODM) das Nações Unidas, com meta de redução em 3/4, entre 1990 e 2015, o que representa valor igual ou inferior a 35 óbitos maternos por 100 mil nascidos vivos no Brasil3. Para que este objetivo fosse atingido, a redução da Taxa de Mortalidade Materna no país deveria ser de 5,5\% ao ano. Para um monitoramento eficaz dos índices de mortalidade materna, com adequada avaliação dos dados disponíveis nos sistemas de informação, calcula-se a taxa de mortalidade materna, podendo ser caracterizada como um indicador sensível da atenção e da qualidade da saúde da mulher ${ }^{4}$.

A fim de realizar um estudo amplo acerca da mortalidade materna no Estado de Alagoas, o objetivo deste trabalho de pesquisa é descrever a epidemiologia da 
mortalidade materna no Estado. Como objetivos específicos, procurou-se analisar a mortalidade materna pelo viés da raça negra e mapear o atendimento às mães parturientes no período de 2006 a 2015.

\section{MÉTODO}

Inicialmente, os óbitos foram selecionados a partir do Sistema de Informação sobre Mortalidade (SIM). Foi escolhida a opção "Tabulação de Dados", em que se obtêm tabelas por variáveis. Em seguida, selecionou-se a opção "Mortalidade 1996-2016 pelo CID-10". Logo após, "Óbito de mulheres em idade fértil e óbitos maternos", selecionando o Estado de Alagoas.

Os óbitos considerados mortalidade materna são aqueles que ocorreram no período de gestação, parto ou puerpério até 42 dias, sendo necessário incluir a informação de que o puerpério é restrito a esse período de tempo5 ${ }^{5}$

De posse dos dados acerca da mortalidade materna, para efeito do cálculo da taxa, foram colhidos os dados de nascidos vivos nos anos de 2006 a 2015.

Os dados serão selecionados a partir do Sistema de Informação sobre Nascidos Vivos (SINASC). Deve-se selecionar a opção "Tabulação de Dados", em que se obtêm tabelas por variáveis.

Devem-se selecionar a opção "Nascidos Vivos - 1994 a 2016" e, em seguida, "Nascidos Vivos" e o Estado de Alagoas. A variável raça foi selecionada de maneira que a população negra corresponde a pretas e pardas.

Com os dados de mortalidade materna e nascidos vivos, foi calculada, para cada ano da série histórica - 2006 a 2015 - a Taxa de Mortalidade Materna.

Para o cálculo, foi empregada a fórmula:

Número de óbitos de mulheres residentes por causas ligadas à gravidez, parto e puerpério em determinado ano Número de nascidos vivos no mesmo ano

Os números de nascidos vivos foram utilizados tanto de forma geral, para a análise de mortalidade dos anos, como também de forma individualizada para cada raça/cor.

Fez-se uso de dados reportados pelo IBGE 2010 sobre características da população do Estado de Alagoas - número de indivíduos por raça/cor e número de mulheres de dez a 49 anos por raça/cor - e calculadas porcentagens das mesmas.

Com as informações das Taxas de Mortalidade Materna da série histórica em 
cada Região de Saúde, foi utilizado o programa Excel para a confecção de tabelas e gráficos que ilustrem a situação da mortalidade materna.

De acordo com a série histórica em cada Região de Saúde, foram confeccionados gráficos e mapas do Estado de Alagoas que ilustrem cada uma das dez Regiões de Saúde e os respectivos valores de taxa com o auxílio do programa TABWIN, baixado diretamente da plataforma on-line do DATASUS.

Foi realizado o cálculo da razão de possibilidades denominado odds ratio. Após essa etapa, calculou-se a probabilidade de ocorrência de óbito dividida pela probabilidade da não ocorrência do mesmo evento.

Sim (raça/cor1): Número de óbitos (raça/cor1)/Número de nascidos vivos (raça/cor1);

Não (raça/cor1): Número de nascidos vivos - número de óbitos (raça/cor1)/Número de nascidos vivos (raça/cor1);

Risco Relativo (raça/cor1): Sim (raça/cor1)/Não (raça/cor1).

\section{RESULTADOS}

No Estado de Alagoas, série histórica de 2006 a 2015, ocorreram 230 óbitos de mulheres entre dez e 49 anos por causa materna em uma média de 23 óbitos anuais. O total de óbitos de mulheres em idade fértil na série histórica foi de 11.703 em que a mortalidade materna corresponde a aproximadamente $2 \%$.

A percentagem de mortes maternas em dez anos foi de $15 \%$ brancas, $80 \%$ pardas e $5 \%$ pretas ( $85 \%$ negras). Ao estimar a raça da população do Estado no período (2006-2015) pelo número de nascidos vivos por raça/cor, 88\% são negros ( $1 \%$ pretos e $87 \%$ pardos) e $12 \%$, brancos. Foi notado que as brancas morreram $15 \%$ para uma população estimada de $12 \%$ e as negras morreram $88 \%$ para uma população estimada de $85 \%$, entretanto, morreram mais brancas nesta análise.

Já em 2010, segundo o IBGE, Alagoas apresentou população de 3.120.494, sendo $31 \%$ brancas, $8 \%$ pretas e $61 \%$ pardas (população negra $69 \%$ ). Ainda segundo o Censo de 2010 (IBGE), das 1.042.247 mulheres de dez a 49 anos, 31\% eram brancas e $62 \%$, negras ${ }^{6}$.

A percentagem de mortes maternas por raça/cor no ano de 2010 foi de $17 \%$ nas brancas, $9 \%$ nas pretas e $74 \%$ nas pardas ( $83 \%$ negras). Em comparação direta, observa-se que, para aquele ano, as percentagens de morte de negras (83\%) foram superiores à constituição racial tanto em sua totalidade (69\%) como restringido em idade fértil (69\%). Já as mulheres brancas obtiveram percentagem 
de morte (14\%) inferior à constituição racial: $31 \%$ da população total e $31 \%$ das mulheres em idade fértil (Tabela 1 ).

\section{Tabela 1. Relação da Porcentagem de Mortes Maternas e a Porcentagem da População por Raça/Cor.}

\begin{tabular}{|c|c|c|c|c|c|}
\hline 忍 & 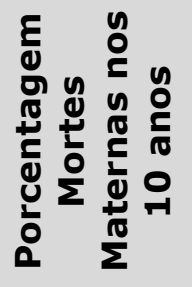 & 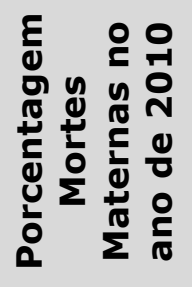 & 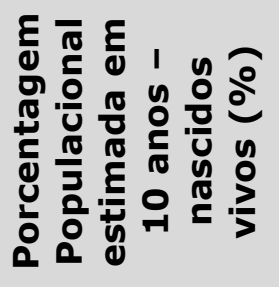 & 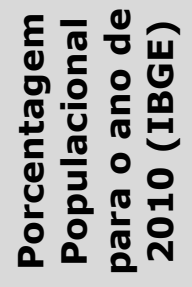 & 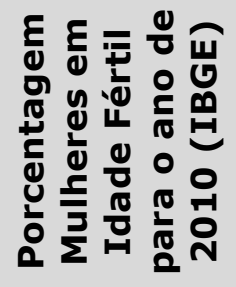 \\
\hline Brancas & $15 \%$ & $17 \%$ & $12 \%$ & $31 \%$ & $31 \%$ \\
\hline Pardas & $80 \%$ & $74 \%$ & $87 \%$ & $61 \%$ & $62 \%$ \\
\hline Pretas & $5 \%$ & $9 \%$ & $1 \%$ & $8 \%$ & $7 \%$ \\
\hline Negras & $85 \%$ & $83 \%$ & $88 \%$ & $69 \%$ & $69 \%$ \\
\hline
\end{tabular}

Fonte: SIM DATASUS/Censo 2010 IBGE/População residente DATASUS/IBGE.

A figura 1 representa a escolaridade relativa à raça/cor das mortes ocorridas no período de dez anos em números absolutos. Ao se analisar a escolarização das mulheres que vieram a óbito por causas maternas, evidencia-se que, quanto mais anos de estudo, menor foi a mortalidade materna: apenas dez óbitos ocorreram em mulheres com escolaridade acima de 12 anos e, por outro lado, houve 24 mortes entre as que não estudaram. Das que não estudaram, todas eram negras. O maior número de óbitos ocorreu entre mulheres que não terminaram o Ensino Fundamental (nenhuma, um a três e quatro a sete anos) - 129 óbitos, cerca de 75\% (Figura 1).

Figura 1. Anos de Escolaridade em Óbitos Durante a Gestação, Parto e Puerpério até 42 Dias Por Raça/Cor.

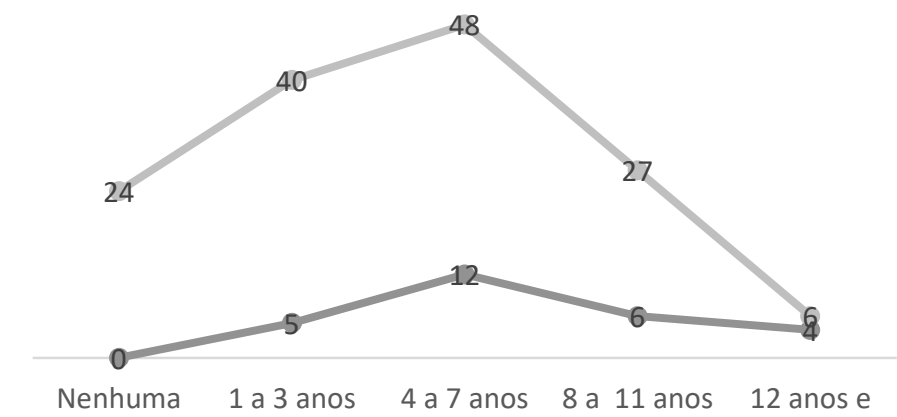

mais

- Branca - Negra 
Fonte: Sistema de Informações sobre Mortalidade (SIM)/Secretaria de Vigilância em Saúde (SVS)/Ministério da Saúde (MS).

Nota-se, analisando a tabela 2, que existe grande variabilidade na taxa de mortalidade de acordo com as Regiões de Saúde. Enquanto a segunda Região de Saúde de Alagoas (formada pelos municípios: Jacuípe, Japaratinga, Maragogi, Matriz de Camaragibe, Passo de Camaragibe, Porto Calvo, Porto de Pedras, São Luís do Quitunde e São Miguel dos Milagres) apresentou as maiores taxas de mortalidade em quatro anos (2007, 2011, 2012 e 2014), cinco regiões não foram as protagonistas da taxa de mortalidade em nenhum dos anos avaliados (1a, $3^{a}$, $\left.5^{a}, 6^{a}, 7^{a}\right)$ (Tabela 2).

Tabela 2. Variação Da Taxa de Mortalidade Materna Por Ano.

\begin{tabular}{ccc}
\hline Ano & $\begin{array}{c}\text { Variação da taxa de mortalidade } \\
\text { materna no Estado de Alagoas } \\
\text { (por 100 mil nascidos vivos) }\end{array}$ & $\begin{array}{c}\text { Região de saúde detentora } \\
\text { da maior taxa naquele ano }\end{array}$ \\
\hline 2006 & $8,71-67,88$ & $8^{a}$ \\
2007 & $18,8-130,89$ & $2^{a}$ \\
2008 & $20,24-116,95$ & $4^{a}$ \\
2009 & $16,29-154,67$ & $4^{a}$ \\
2010 & $28,28-150,6$ & $9^{a}$ \\
2011 & $29,56-344,03$ & $2^{a}$ \\
2012 & $15,95-363,19$ & $2^{a}$ \\
2013 & $24,26-173,91$ & $10^{a}$ \\
2014 & $36,91-432,9$ & $2^{a}$ \\
2015 & $36,91-170,06$ & $10^{a}$ \\
\hline
\end{tabular}

Fonte: MS/SVS/DASIS - Sistema de Informações sobre Nascidos Vivos - SINASC. MS/SVS/CGIAE Sistema de Informações sobre Mortalidade - SIM.

Ao se analisar a figura 2, elaborado a partir das taxas de mortalidade materna por Região de Saúde no Estado de Alagoas na série histórica 2006 - 2015, observa-se que, no período, as Regiões de Saúde com maior taxa foram: $3^{a}$ (50) e $10^{a}$ (49). Quatro Regiões de Saúde apresentaram taxa de mortalidade inferior a 35, sendo elas: 7a, 4a $1^{a}$ e $6^{a}$. A $8^{a}$ Região de Saúde obteve taxa entre 35 e 39. A $2^{a}$ e $9^{a}$ tiveram taxa entre 43 e 46.

Ainda, ao se dividir entre as séries 2006 - 2010 e 2011 - 2015, as Regiões de Saúde com maior taxa de mortalidade na primeira série foram: $8^{a}(36)$, $6^{a}$ (33) e $4^{a}$ região (31). Já entre 2011 - 2015, as regiões em destaque foram: $3^{a}$ (84), $10^{\mathrm{a}}(82)$ e $2^{\mathrm{a}}(66)$ (Figura 2 ). 
Figura 2. Taxa de Mortalidade Materna por 100 Mil Nascidos Vivos por Região de Saúde.

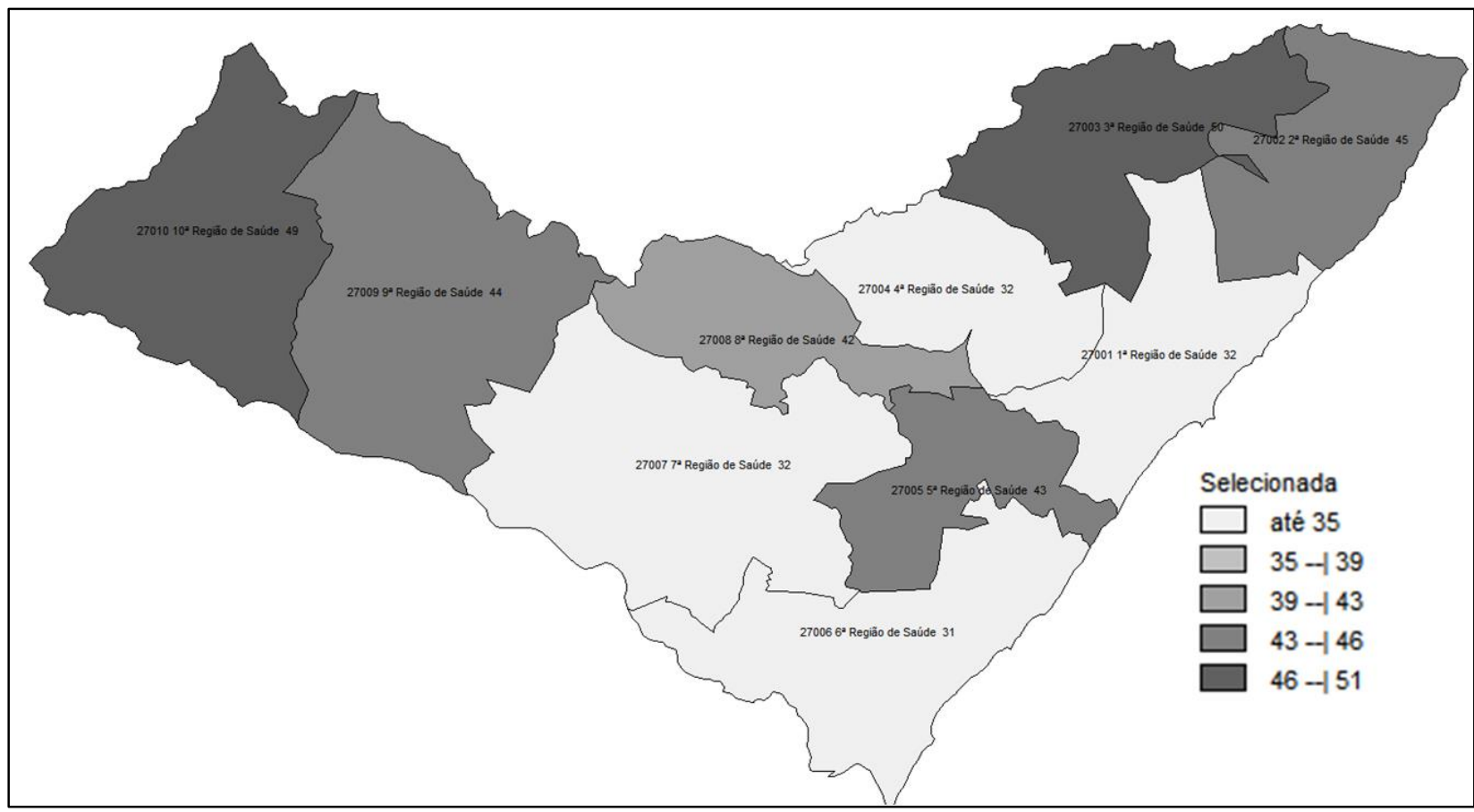

Fonte: MS/SVS/DASIS - Sistema de Informações sobre Nascidos Vivos - SINASC. MS/SVS/CGIAE Sistema de Informações sobre

Na tabela 3, pode-se notar o cálculo do risco de morte pelo odds ratio no segmento de cada raça/cor em que as brancas obtiveram 0,359; pardas, 1,591 e negras, 1,561. A cor preta, apesar de toda análise percentual realizada, neste estudo, teve associação não significativa. Para tornar significativa esta associação, dever-se-ia aumentar o número da população: incluindo mais anos ou incluindo outros Estados ou regiões do país.

A cor branca não é fator de risco, mas, sim, de proteção, corroborando a análise realizada para o ano de 2010 e o percentual de morte em brancas para o mesmo ano. A cor parda e a população negra são fatores de risco (Tabela 3). 
Tabela 3. Análise De Risco Pelo ODDS Segundo Categorias De Raça/Cor.

\begin{tabular}{|c|c|c|c|c|c|c|c|c|c|}
\hline \multirow{3}{*}{ Raça/Cor } & \multicolumn{6}{|c|}{ Óbito Materno } & \multirow{3}{*}{ ODDS } & \multicolumn{2}{|c|}{ IC $95 \%$} \\
\hline & \multicolumn{2}{|r|}{ Sim } & \multicolumn{2}{|c|}{ Não } & \multicolumn{2}{|c|}{ Total } & & \multirow[t]{2}{*}{ L.Inf. } & \multirow[t]{2}{*}{ LSup. } \\
\hline & $\mathbf{n}$ & $\%$ & $\mathbf{n}$ & $\%$ & $\mathbf{N}$ & $\%$ & & & \\
\hline \multicolumn{10}{|l|}{ Branca } \\
\hline Sim & 32 & 0,0010 & 3265776 & 99,9990 & 3265808 & 31,02 & & & \\
\hline Não & 198 & 0,0027 & 7260568 & 99,9973 & 7260766 & 68,98 & 0,359 & 0,247 & 0,522 \\
\hline Total & 230 & 0,0022 & 10526344 & 99,9978 & 10526574 & 100,00 & & & \\
\hline \multicolumn{10}{|l|}{ Preta * } \\
\hline Sim & 11 & 0,0017 & 644099 & 99,9983 & 644110 & 6,12 & & & \\
\hline Não & 219 & 0,0022 & 9882245 & 99,9978 & 9882464 & 93,88 & 0,771 & 0,421 & 1,412 \\
\hline Total & 230 & 0,0022 & 10526344 & 99,9978 & 10526574 & 100,00 & & & \\
\hline \multicolumn{10}{|l|}{ Parda } \\
\hline Sim & 164 & 0,0026 & 6418181 & 99,9974 & 6418345 & 60,97 & & & \\
\hline Não & 66 & 0,0016 & 4108163 & 99,9984 & 4108229 & 39,03 & 1,591 & 1,195 & 2,117 \\
\hline Total & 230 & 0,0022 & 10526344 & 99,9978 & 10526574 & 100,00 & & & \\
\hline \multicolumn{10}{|l|}{ Negra } \\
\hline Sim & 175 & 0,0025 & 7062280 & 99,9975 & 7062455 & 67,09 & & & \\
\hline Não & 55 & 0,0016 & 3464064 & 99,9984 & 3464119 & 32,91 & 1,561 & 1,153 & 2,113 \\
\hline Total & 230 & 0,0022 & 10526344 & 99,9978 & 10526574 & 100,00 & & & \\
\hline
\end{tabular}

Fonte: SIM DATASUS/Censo 2010 IBGE/População residente DATASUS/IBGE. Processamento: autores. Nota * Não significativo

\section{DISCUSSÃO}

As porcentagens de morte da série histórica total, quando comparadas aos nascidos vivos do período, levam a crer que, embora as negras apresentem maior número de mortes em números absolutos, elas também estão em número elevado na população. No advento em que se estimou a população pelo número de nascidos vivos, as mulheres negras, que compreendem pardas e pretas, morreram menos do que seu contingente populacional. Isto quer dizer que, em relação às mulheres brancas, estas morreram mais. Já quando analisados os números somente para o ano de 2010, as mulheres negras morreram em percentagem maior que a sua 
população, proporcionalmente, enquanto as brancas vieram a óbito $14 \%$ menos do que seu contingente populacional.

As mulheres pretas, por serem em menor número quando comparadas às brancas e pardas, apresentam taxa de mortalidade bastante elevada nos anos em que existem ocorrências de morte desse seguimento. Nesta análise, evidencia-se que as pretas, quando analisadas estratificadamente e relativizadas pelos nascidos vivos do mesmo grupo, chegaram a apresentar taxa de mortalidade 900 vezes maior que as pardas e brancas.

Apesar disso, na tabela 3, foi salientado o maior risco de morte da população negra em relação à população branca. Diferentemente da análise feita na tabela 1 , relativa a todos os anos da série histórica em que as brancas morreram em porcentagem maior que a sua população estimada pelos nascidos vivos, no estudo odds ratio, pode-se salientar que a população negra possui maior chance de morte e o componente dessa população que acrescenta maior risco é a parda, considerando que a preta não teve análise significativa.

O crescimento da Taxa de Mortalidade Materna de todas as raças ocorreu principalmente no segundo quinquênio (2010-2015), a despeito da criação da Rede Cegonha, programa que visa ao direito ao planejamento reprodutivo e atenção humanizada à gravidez, em 2010. Contudo, quando avaliadas somente as mulheres pretas, visualiza-se que esta é a única raça em que se diminuiu a taxa de mortalidade em relação ao quinquênio anterior (2006-2010).

Isso, possivelmente, pode estar atrelado à criação da Política Nacional de Saúde Integral da População Negra (PNSIPN), em 2007, e protagonizada a partir 2009, que visa à melhoria das condições de saúde desta população7.

No momento em que a questão da raça/cor é um elemento autodeclarável, ou seja, definido pelos parentes da vítima no caso do óbito, pela mãe no caso de nascidos vivos e vulneráveis e pelo próprio indivíduo, assim como a falta de características próprias e exclusivas para se definir a população, principalmente parda, pelo caráter multimiscigenado, torna-se a raça uma variável oscilante e, às vezes, de difícil definição.

A análise pode também estar sendo dificultada ao se considerar o número de casos ignorados - preenchimento inadequado da variável raça/cor e a fidedignidade dos dados relacionados ao tempo puerperal -, que obteve número considerável ao redor dos anos - 23 óbitos, $10 \%$ dos óbitos totais, chegando a sete no ano de 2014, e também devido ao aumento das notificações, registros e 
informatização de mortes, em função da expansão tecnológica, que automaticamente elevam o número de mortes registradas no decorrer dos anos.

A figura 1 apresenta-se díspar em relação à raça/cor em quase toda sua apresentação, estando a morte das mulheres negras sempre em elevados patamares quando analisados os menores índices de escolaridade.

Contudo, avaliando somente a variável que contém a maior escolaridade (12 ou mais anos), o número de mortes entre as raças aproxima-se. Neste momento, as mulheres negras apresentam pequena discrepância em números absolutos em relação às mulheres brancas, um fator considerável quando comparado à própria constituição racial do Estado de Alagoas e, também, à falta de escolaridade proporcional da população negra no Estado. Além disso, o achado reforça o fato de que a escolaridade pode ser um fator protetor/preventivo da mortalidade materna.

Assim, a maior proporção de mortes das negras está atrelada a questões como condições sociais (assim como escolaridade) e também ao grande contingente da população negra no Estado, o que aumenta a demanda de mulheres negras nos serviços e ocasiona mais mortes dessa raça, e, novamente, à dificuldade de melhor análise pelo grande número de casos ignorados.

Por meio de análises realizadas nas diferentes Regiões de Saúde de Alagoas, foram percebidas importantes variações nas Taxas de Mortalidade Materna, o que reflete as discrepâncias para cada Região de Saúde, principalmente em função das diferenças populacionais de cada município e pelas dificuldades de acesso aos hospitais regionais e a um pré-natal eficiente.

A $2^{a}$ Região de Saúde, apesar de ter maior número de anos com a maior taxa de mortalidade do Estado (2007, 2011, 2012, 2014), dispõe para atendimentos de média e alta complexidade os hospitais de Porto Calvo, de Matriz de Camaragibe e Hospital José Augusto, em São Luís do Quitunde, além de utilizar os serviços do Hospital Geral do Estado, em Maceió.

Apesar de essa região dispor de hospitais de média e alta complexidade, a taxa de mortalidade continua elevada. Esse dado reflete a necessidade de ações eficientes voltadas à redução da mortalidade materna. São necessários maiores investimentos na atenção básica e em serviços de atenção à gestante, uma vez que a maioria dos municípios utiliza, como referência, as maternidades de Maceió, distantes, portanto, da residência de muitas mães. Ressalta-se, ainda, a 
necessidade de implementar políticas de investigação do óbito, que auxiliam na busca pelo melhoramento dos serviços ofertados às parturientes.

Apesar do aumento dos valores se justificar, entre outros, pelo maior número de registros, as taxas continuam altas, muito além do considerado ideal pela ONU (taxa de mortalidade menor que 35), o que reflete a necessidade de políticas eficazes que possam romper as iniquidades presentes e fornecer às mães um atendimento pré-natal eficiente, assim como a disponibilidade de leitos maternos próximos às suas residências para um atendimento ágil.

\section{CONCLUSÃO}

Foi notado que a análise das mulheres negras apresenta discrepâncias em relação aos seus componentes (entre pretas e pardas). Enquanto as pardas apresentaram-se praticamente com números equivalentes às brancas na maioria das análises (exceto odds ratio, em que as pardas tiveram maior risco), as negras, nos anos em que existiram óbitos, tiveram índice de mortalidade bastante superior às demais (apesar de, na análise odds ratio, haver avaliação não significativa), assim como tiveram número de mortes superior proporcionalmente à sua existência na população.

Apesar disso, as mulheres pretas foram as únicas que diminuíram a taxa de mortalidade no segundo quinquênio. Essa diminuição pode estar atrelada à implementação da Política Nacional de Saúde Integral da População Negra, em 2007.

Em relação à escolaridade, a morte das mulheres negras sempre tem elevados patamares, principalmente quando analisados os menores índices de escolaridade. Quanto maior a escolaridade, menores os índices de mortalidade e maior é a aproximação entre os índices de mortalidade entre mulheres brancas e negras. Diante disso, a escolaridade pode estar sendo um fator de proteção.

Quanto à territorialidade do Estado, a 2a Região de Saúde teve a maior taxa de mortalidade nos anos analisados (2007, 2011, 2012, 2014), apesar de dispor de hospitais de média e alta complexidade. Isso pode estar relacionado a uma ineficiência dos serviços quando considerada a atenção às gestantes.

A melhoria dos meios tecnológicos para notificação evidenciou um aumento do número de registros e, consequentemente, a maior qualidade de dados, culminando em um aumento geral dos números no segundo quinquênio dos anos analisados. 
A análise foi dificultada pelo elevado número de casos ignorados, levando à subestimação dos números, e pela falta de características objetivas que definam a raça da população.

\section{REFERÊNCIAS}

1. Lopes F, Buchalla CM, Ayres JRCM. Black and non-black women and vulnerability to HIV/AIDS in São Paulo, Brazil. Rev Saúde Pública. 2007 Dec; 41(2):39-46. DOI: $10.1590 /$ S0034-89102007000900008

2. Ministério da Saúde (BR), Secretaria de Atenção à Saúde, Departamento de Ações Programáticas Estratégicas. Perspectiva da Equidade no Plano Nacional pela redução da mortalidade materna e neonatal: atenção à saúde das mulheres negras [Internet]. Brasília: Ministério da Saúde; 2005 [cited 2019 Aug 15]. Available from: em: http://bvsms.saude.gov.br/bvs/publicacoes/perspectiva_equidade_pacto_naciona I.pdf

3. Ministério da Saúde (BR). Secretaria de vigilância em Saúde. Mortalidade materna no Brasil. Bol Epidemiol [Internet]. 2012 [cited 2019 Oct 21]; 43(1):1-7. Available from: https://portalarquivos2.saude.gov.br/images/pdf/2014/julho/23/BE-2012-43--1--pag1-a-7---Mortalidade-Materna.pdf

4. Ministério da Saúde (BR). Pacto nacional pela redução da mortalidade materna e neonatal. Informe Atenção Básica [Internet]. 2004 May/June [cited 2017 Aug 15]; 5(22):1-2. Available from: http://portal.saude.gov.br/portal/saude/odm_saude/visualizar_texto.cfm?idtxt=35197

5. Organização Mundial de Saúde. Classificação Internacional de Doenças: décima revisão (CID-10). 4th ed. São Paulo: EDUSP; 1998.

6. Ministério do Planejamento, Orçamento e Gestão (BR), Instituto Brasileiro de Geografia e Estatística. Censo demográfico 2010 [Internet]. Rio de Janeiro: IBGE; 2010 [cited 2019 Sep 15]. Available from: https://censo2010.ibge.gov.br/resultados.html

7. Ministério da Saúde (BR), Secretaria Especial de Políticas de Promoção da Igualdade Racial. Política Nacional de Saúde Integral da População Negra [Internet]. Brasília: Ministério da Saúde; 2007 [cited 2019 Apr 15]. Available from: http://bvsms.saude.gov.br/bvs/publicacoes/politica_nacional_saude_populacao_negra .pdf 